\title{
WBRT Plus SIB With Image Guided Intensity-Modulated Radiotherapy for Multiple Brain Metastases
}

\author{
Tiziana Bruno*, Antonella Mazzonello and Ivan Fazio \\ Casa Di Cura Macchiarella, University of Study of Palermo, Italy
}

Submission: September 04, 2018; Published: September 26, 2018

"Correspondence Address: Tiziana Bruno, Casa Di Cura Macchiarella, University of Study of Palermo, Piazza Marina, 61 - Palermo, Italy, Email: brtiziana@libero.it

\section{Introduction}

$20 \%$ up to $40 \%$ of cancer patients develop brain metastases during the course of their illness. Up to $70 \%$ of cases, these multiple brain metastases. The prognosis is poor, with a median survival of 7.1 months for the class to better prognosis according to Recursive Partitioning Analysis (RPA) and 11 months according Graded Prognostic Assessment (GPA). The Whole Brain Irradiation (WBRT) is the primary treatment option for patients with multiple brain metastases. However, after this treatment, the rate of local failure and distance can occur in a substantial number of patients. Several authors have shown that the Intensity Modulated Radiotherapy (IMRT), both static and dynamic, providing adequate conformity of dose distribution and fast delivery times, allows the use of WBRT technique with Simultaneous Integrated Boost (SIB) by regions of macroscopic tumor in most cases, including scenarios palliatives such as the treatment of brain metastases. The endpoints of this retrospective study were to evaluate the local control intracranial Lesions Treated Brain (LTC), Progression-Free Survival intracranial (PFS), Overall Survival (OS) and acute and late toxicity (CTCAE v4.0).

\section{Methods}

From July 2016 to January 2017, 13 patients were treated according to protocol WBRT with SIB for brain metastases. Eleven patients with multiple brain metastases and two patients with single brain metastasis is not susceptible of surgery or radiosurgery, per location, size and life expectancy of no more than four months according to RPA. The eligibility of patients for this study retroprospective was: history of histologically confirmed cancer, brain metastases diagnosed at Computed Tomography (CT) and / or Magnetic Resonance Imaging (MRI) with contrast medium (mdc) pretreatment, age $\leq 85$ years, any Karnofsky Performance Status (KPS), controlled primary tumor, no previous cranial irradiation. The median age of patients at diagnosis of brain lesions was 51 years (range 32-85 years). Four patients metastatic disease brain originated from a lung adenocarcinoma, for four more patients from a melanoma, for three from a carcinoma of the breast, for a patient from a small cell and for another patient it was not possible to determine the primary tumor. The average number of brain lesions treated per patient was 1.85 (range 1-4); the choice of such brain lesions treated, depended on their size, presence of symptoms and the same distance of at least $5 \mathrm{~mm}$ from the optical structures and the brain stem. Most brain lesions were localized in the parietal lobe $(25 \%)$.

Six patients extracranial disease at the time of radiation treatment. Two patients had previously undergone brain metastasectomy for other injuries. The patients were positioned supine and have performed a simulation CT without contrast agent with scans with a pitch of $3 \mathrm{~mm}$, after packaging of immobilisation system with mask thermoplastic custom. The volumes of irradiation were defined according to the report of the International Commission Radiation Units (ICRU). The Gross Tumor Volume (GTV), represented by metastatic disease, has been defined on the basis of the fusion of MRI scans with gadolinium diagnostics on T1 and / or T2-weighted images with the images obtained by CT centering. The Planning Target Volume (PTV) for each metastases was obtained by adding a margin of $2 \mathrm{~mm}$ to GTV. The planning target volume for the whole brain has been obtained from the PTV outline the whole brain. The organs at risk included the scalp, eyes and clear bilaterally. For all patients, in combination with WBRT 30 Gy in 10 fractions over the entire brain, it was administered a SIB of 10 Gy in 10 fractions over major brain lesions. The cumulative dose has been dispensed and then 40 Gy in 10 fractions.

Prescription all'isodose of $100 \%$. The treatment plan is developed with the planning system Oncentra ${ }^{\circledR}$ External Beam using a volumetric IMRT technique with two arches (VMAT) $\left(185^{\circ}-175^{\circ}\right.$ and $\left.175^{\circ}-185^{\circ}\right)$ for 5 patients and a static IMRT technique to 6 multiple fields (OAD 310, LLD 270, OPD 230, OAS 50, LLS 90 and OPS 130) for the remaining 8 patients, dispensed with Synergy linear accelerator (Elekta) with photon beams of 6 MV, with Millennium Multileaf Collimator (MLC) with 80 blades. 


\section{Cancer Therapy \& Oncology International Journal}

All patients were subjected to verification set-up and possible correction of the errors of set up, prior to treatment. Steroid therapy with dexamethasone was administered to all patients for the duration of the treatment; in addition to this, for symptomatic patients, it was necessary to adjust your therapy depletional practiced, with drugs such as mannitol infusion.

\section{Results}

Thirteen patients (24 metastatic brain lesions) were treated. The median follow-up was 10.8 months (range, 2-20 months) and performed with clinical examination and imaging studies (contrast-enhanced CT and / or MRI with gadolinium) repeated at least six weeks and then every three to four months of completion radiation treatment. Seven patients had intracranial local tumor control (LTC at six months and one year amounted to $91.7 \%$ and $62.9 \%$, respectively). Four patients died within five months after the end of radiation therapy for local disease progression and / or remotely. Two patients had the appearance of a new brain lesion, respectively, to 10 and 14 months after the end of radiotherapy; the first patient has chemotherapy performed with partial remission of new lesions and the absence of clinical disorders of note, the second patient stopped chemotherapy after one cycle to severe weight loss and past general clinical condition.

The intracranial Progression-Free Survival rates (PFS) at six months and one year was estimated to be $90.9 \%$ and $81.8 \%$, respectively. A patient with multiple brain metastases from breast cancer is in complete remission to ten months after the end of radiotherapy and chemotherapy makes no medical conditions of note, apart from acne-like rash. Overall Survival (OS) to one year amounted to $69.2 \%$. The acute and late toxicity was mild to moderate (G1-G2, according to CTCAE v4.0) and included transient disturbances such as alopecia, fatigue, nausea, headache and dizziness subjective that has been practiced appropriate supportive care. Two patients experienced mental confusion. Five patients did not experience any side effects radio-induced throughout the duration of the treatment and in the subsequent follow-up. A patient with impaired vision and balance at the time of diagnosis, had an improvement of the above symptoms already under treatment. A patient with three brain metastases from melanoma and extracranial disease and pelvic lymph node, was suspended after three treatment sessions to deterioration of overall clinical condition. No patient developed radiation necrosis and / or leuco-encephalopathy by the end of radiotherapy.

\section{Discussion}

The literature is full of works, all agree that treatment with WBRT SIB allows to achieve good therapeutic results without severe toxicity. Oehlke et al. [1] evaluated 20 patients with multiple metastases (2-13 brain metastases) treated with WBRT (30 Gy in 12 fractions) with SIB (51 Gy) using VMAT to 2-4 arches, with rate of LTC, PFS and OS to one year by $73 \%, 45.3 \%$ and $60 \%$ respectively, and no acute toxicity and / or late grade than G2. Zhou et al. [2] have described the treatment WBRT with IG-IMRT SIB more tolerable and effective for 29 patients with multiple brain metastases from NSCLC, and LTC reported a rate of $62.9 \%$, while $41.4 \%$ was both the PFS that the median OS to one year; 3 of 87 lesions showed radiation necrosis. $55.1 \%$ was the overall survival rate at six months reported by Weber et al. [3] of 29 patients with multiple metastases and VMAT treatment (WBRT 30 Gy, 40 Gy in 10 fractions SIB) with no significant toxicity.

\section{Conclusion}

The protocol WBRT with SIB has detected feasible and safe. All patients, except one, have completed treatment without severe toxicity. The follow-up has shown encouraging results in terms of LTC, PFS and OS.

\section{References}

1. Oehlke O, Wucherpfennig D, Fels F, Frings L, Egger K, et al. (2015) Whole brain irradiation with hippocampal sparing and dose escalation on multiple brain metastases: Local tumour control and survival. Strahlenther Onkol 191(6): 461-469.

2. Zhou L, Liu J, Xue J, Xu Y, Gong Y, et al. (2014) Whole brain radiotherapy plus simultaneous in-field boost with image guided intensitymodulated radiotherapy for brain metastases of non-small cell lung cancer. Radiat Oncol 9: 117.

3. Weber DC, Caparrotti F, Laouiti M, Malek K (2011) Simultaneous infield boost for patients with 1 to 4 brain metastasis/es treated with volumetric modulated arc therapy: a prospective study on quality-oflife. Radiotion Oncol 6: 79.

\section{Your next submission with Juniper Publishers will reach you the below assets}

- Quality Editorial service

- Swift Peer Review

- Reprints availability

- E-prints Service

- Manuscript Podcast for convenient understanding

- Global attainment for your research

- Manuscript accessibility in different formats ( Pdf, E-pub, Full Text, Audio)

- Unceasing customer service

Track the below URL for one-step submission https://juniperpublishers.com/online-submission.php 